\title{
Periodic boundary value problems for first order dynamic equations on time scales
}

\author{
Xiaozhi Zhang and Chuanxi Zhu*
}

* Correspondence: chuanxizhu@126.com Department of Mathematics, Nanchang University, Nanchang, 330031, People's Republic of China

\section{Abstract}

In this work, the existence criteria of extremal solutions of periodic boundary value problems for the first-order dynamic equations on time scales are given by using the method of lower and upper solutions coupled with the monotone iterative technique. Our results generalize and improve some existing results. Two examples are provided to show the effectiveness and feasibility of the obtained results.

Keywords: Periodic boundary value problems, time scales, lower and upper solutions

\section{Introduction}

Recently, the theory of impulsive differential equations has been investigated extensively in simulating processes and phenomena subject to short-time perturbations during their evolution, moreover, such equations have a tremendous potential for applications in biology, physics, epidemic models, engineering, ect. (see [1-4]). Especially, the study of impulsive dynamic equations on time scales has also attracted much attention since it provides an unifying structure for differential equations in the continuous cases and the finite difference equations in the discrete cases, see [5-17] and references therein. Most of them were devoted to the existence of solutions for periodic boundary value problems (PBVP) by means of some fixed point theorems [18-22] such as the Tarski's fixed point theorem [17], Guo-Krasnoselskii fixed-point theorem [18], and twin fixed-point theorem in a cone [19], etc..

Li et al. [4] considered the following periodic boundary value problem with impulses

$$
\left\{\begin{array}{l}
u^{\prime}(t)=g(t, u(t), u(\theta(t))), \quad t \in J=[0, T], \quad t \neq t_{k} \\
\Delta u\left(t_{k}\right)=I_{k}\left(u\left(t_{k}\right)\right), \quad k=1,2, \ldots, p, \\
u(0)=u(T)
\end{array}\right.
$$

where $0=t_{0}<t_{1}<t_{2}<\ldots<t_{p}<t_{p+1}=T, J_{0}=\lambda\left\{t_{1}, \ldots, t_{p}\right\}, g \in C\left(J \times R^{2}, R\right)$, and $\theta \in C(J$, $R), 0 \leq \theta(t) \leq t, t \in J, \Delta u\left(t_{k}\right)=u\left(t_{k}^{+}\right)-u\left(t_{k}\right)$.

In [6], the authors discussed the following periodic boundary value problem by using the upper and lower solution method and monotone iterative technique

$$
\left\{\begin{array}{l}
\gamma^{\Delta}(t)=f(t, \gamma(t)), \quad t \in J:=[0, T] \cap \mathbf{T}, \quad t \neq t_{k}, k=1,2, \ldots, m \\
\operatorname{Imp}(\gamma)\left(t_{k}\right):=I_{k}\left(\gamma\left(t_{k}^{-}\right)\right), \quad k=1,2, \ldots, m, \\
y(0)=\gamma(\sigma(T)) .
\end{array}\right.
$$

\section{Springer}

(c) 2012 Zhang and Zhu; licensee Springer. This is an Open Access article distributed under the terms of the Creative Commons Attribution License (http://creativecommons.org/licenses/by/2.0), which permits unrestricted use, distribution, and reproduction in any medium, provided the original work is properly cited. 
Although, the existence of extremal solutions to equation (1.2) was studied in [6] by using the outlined method, the results were obtained only under the case: $\alpha \leq \beta$, where $\alpha$ and $\beta$ are the lower and upper solutions of PBVP (1.2), respectively. To the best of our knowledge, while using the method of lower and upper solutions, one can consider the problem under another case: $\alpha \geq \beta$, the readers can refer to [12,23-25] and references therein. For example, in [23], He and Zhang analyzed the existence for the extremal solutions to first order impulsive difference equations with periodic boundary conditions by utilizing the monotone iterative technique under these two cases. However, there are few papers to deal with the existence for the extremal solutions to periodic boundary value problems of first order dynamic equations on time scales based on the method of lower and upper solutions coupled with monotone iterative technique under these two cases. Thus, in this paper, we make the first attempt to consider the following periodic boundary value problem of first order dynamical equation on time scales:

$$
\left\{\begin{array}{l}
y^{\Delta}(t)+p(t) y^{\sigma}(t)=f\left(t, y^{\sigma}(t)\right), \quad t \in J:=[0, T] \cap \mathbf{T}, \quad t \neq t_{k}, k=1,2, \ldots, q, \\
\Delta y\left(t_{k}\right)=I_{k}\left(y\left(t_{k}^{-}\right)\right), \quad k=1,2, \ldots, q, \\
y(0)=y(\sigma(T)),
\end{array}\right.
$$

where $\mathbf{T}$ is a time scale, $T>0$ is fixed. $f \in C(J \times R, R), I_{k} \in C(R, R), p:[0, T]_{\mathbf{T}} \rightarrow[0$, $\infty)$ is rd-continuous, $t_{k} \in[0, T] \cap \mathbf{T}$ and $0<t_{1}<\ldots<t_{q}<T . \Delta y\left(t_{k}\right)=\gamma\left(t_{k}^{+}\right)-\gamma\left(t_{k}^{-}\right)$, $\gamma\left(t_{k}^{+}\right)$and $\gamma\left(t_{k}^{-}\right)$denote right and left limits of $y(t)$ at $t=t_{k}$ in the sense of time scales respectively, that is, $t_{k}+h \in[0, T] \cap \mathbf{T}$ for each $h$ in a neighborhood of $0 . \sigma$ is a function that will be defined later and $y^{\sigma}(t)=y(\sigma(t))$. The aim of this paper is to obtain the existence criteria of extremal solutions of PBVP (1.3) under the two cases expressed above. Our results extend and generalize the results of Geng et al. [6].

The remainder of this paper is organized as follows. Some preliminary definitions, lemmas and notations on time scales are given in Section 2. In Sections 3 and 4, by means of lower and upper solutions and monotone iterative technique, the existence of extremal solutions for PBVP (1.3) is proved under two cases: the lower and upper solutions with well-order and with reverse-order, respectively. Finally, two examples are simplified to illustrate the effectiveness and feasibility of the obtained results.

\section{Preliminaries}

In this section, we state some fundamental definitions and results on time scales, we refer readers to [5-11].

Let $\mathbf{T}$ be a nonempty closed subset (time scale) of $R$. We denote the forward and backward jump operators $\sigma, \rho: \mathbf{T} \rightarrow \mathbf{T}$ as follows:

$$
\sigma(t)=\inf \{s \in \mathbf{T} \mid s>t\} \quad \text { and } \quad \rho(\mathrm{t})=\sup \{\mathrm{s} \in \mathrm{T} \mid \mathrm{s} ; \mathrm{t}\} .
$$

A point $t \in \mathbf{T}$ is called right-scattered, right-dense, left-scattered, left-dense respectively if $\sigma(t)>t, \sigma(t)=t, \rho(t)<t, \rho(t)=t$ holds. If $\mathbf{T}$ has a right-scattered minimum $m$, then $\mathbf{T}_{k}=\mathbf{T} \backslash\{m\}$; otherwise $\mathbf{T}_{k}=\mathbf{T}$. If $\mathbf{T}$ has a left-scattered maximum $M$, then $\mathbf{T}^{k}$ $=\mathbf{T} \backslash\{M\}$; otherwise $\mathbf{T}^{k}=\mathbf{T}$.

A function $f: \mathbf{T} \rightarrow R$ is called right-dense continuous provided it is continuous at every right-dense points in $\mathbf{T}$ and its left-side limits exist at every left-dense points in $\mathbf{T}$, write $f \in C_{r d}(\mathbf{T})=C_{r d}(\mathbf{T}, R)$.

Definition 2.1. For $x: \mathbf{T} \rightarrow R$ and $t \in \mathbf{T}^{k}$, we define the delta derivative of $x(t), x^{\Delta}(t)$, to be the number (when it exists) with the property that, for any $\varepsilon>0$, there is a 
neighborhood $U$ of $t$ such that

$$
\left|[x(\sigma(t))-x(s)]-x^{\Delta}(t)[\sigma(t)-s]\right|<\varepsilon|\sigma(t)-s|, \text { for all } s \in U .
$$

Remark 2.1. If $\mathbf{T}=R, x^{\Delta}(t)=x^{\prime}(t)$ is the ordinary derivative, and if $\mathbf{T}=Z, x^{\Delta}(t)=x(t$ $+1)-x(t)$ is the usual forward difference operator.

Definition 2.2. $F$ is called an antiderivative of a function $f$ defined on $\mathbf{T}$ if $F^{\Delta}(t)=f(t)$ holds on $\mathbf{T}^{k}$, then the delta integral is defined by

$$
\int_{a}^{t} f(s) \Delta s=F(t)-F(a) .
$$

Lemma 2.1 ([9]). If $f^{\Delta} \geq 0$, then $f$ is increasing.

Lemma 2.2 ([9]). Assume that $f, g: \mathbf{T} \rightarrow R$ are delta differentiable at $t$, then

$$
(f g)^{\Delta}(t)=f^{\Delta}(t) g(t)+f(\sigma(t)) g^{\Delta}(t)=g^{\Delta}(t) f(t)+g(\sigma(t)) f^{\Delta}(t) .
$$

Definition 2.3. A function $p: \mathbf{T} \rightarrow R$ is called regressive if $1+\mu(t) p(t) \neq 0$ for all $t \in$ $\mathbf{T}$, where $\mu(t)=\sigma(t)-t$, which is said to be the graininess function.

Definition 2.4. If $p$ is a regressive function, then the generalized exponential function $e_{p}$ is defined by

$$
e_{p}(t, s)=\exp \left\{\int_{s}^{t} \xi_{\mu(\tau)}(p(\tau)) \Delta \tau\right\}, \text { for } s, t \in \mathbf{T}
$$

with the cylinder transformation

$$
\xi_{h}(z)= \begin{cases}\frac{\log (1+h z)}{h}, & \text { if } h \neq 0, \\ z, & \text { if } h=0,\end{cases}
$$

Let $p, q: T \rightarrow R$ be two regressive functions, we define

$$
p \oplus q=p+q+\mu p q, \quad \ominus p:=-\frac{p}{1+\mu p}, \quad p \ominus q:=p \oplus(\ominus q) .
$$

Then, the exponential function has some properties as follows.

Lemma 2.3 ([9]). Assume that $p, q: \mathbf{T} \rightarrow R$ are two regressive functions, then

(1) $e_{0}(t, s) \equiv 1, e_{p}(t, t) \equiv 1$;

(2) $e_{p}(t, s)=\frac{1}{e_{p}(s, t)}=e_{\ominus p}(s, t)$;

(3) $e_{p}(t, u) e_{p}(u, s)=e_{p}(t, s)$;

(4) $e_{p}^{\Delta}\left(t, t_{0}\right)=p(t) e_{p}\left(t, t_{0}\right)$ for $t \in T^{k}, t_{0} \in T$.

\section{Well-ordered lower and upper solutions}

In this section, we prove the existence theorem of extremal solutions for periodic boundary value problem of first-order dynamic equations on time scales under the case of $\alpha \leq \beta$, where $\alpha$ and $\beta$ are lower and upper solutions of PBVP (1.3).

We assume for the remainder of this paper that the impulsive points $t_{k}, k=1,2, \ldots, q$ are right dense, other cases may be considered similarly. Define the following space

$$
\begin{aligned}
& P C=\left\{y:[0, \sigma(T)] \rightarrow R \mid y_{k} \in C\left(J_{k}, R\right), k=1,2, \ldots, q \text { and there exist } \mathrm{y}\left(\mathrm{t}_{\mathrm{k}}^{+}\right) \text {and } \mathrm{y}\left(\mathrm{t}_{\mathrm{k}}^{-}\right)\right. \\
& \left.\quad \text { with } \mathrm{y}\left(\mathrm{t}_{\mathrm{k}}^{-}\right)=\mathrm{y}\left(\mathrm{t}_{\mathrm{k}}\right), \mathrm{k}=1,2, \ldots, \mathrm{q}\right\},
\end{aligned}
$$


which is a Banach space with the norm $\|y\|_{P C}=\max \left\{\left\|y_{k}\right\|_{J_{k}}, k=0,1, \ldots, q\right\}$, where $y_{k}$ is the restriction of $y$ to $J_{k}=\left(t_{k}, t_{k+1}\right] \subset\left(0, \sigma(T)_{\mathrm{T}}\right], k=1,2, \ldots, q$ and $J_{0}=\left[0, t_{1}\right], t_{q+1}=\sigma(T)$.

Definition 3.1. A function $y \in P C \cap C^{1}\left(\lambda\left\{t_{1}, t_{2}, \ldots, t_{q}\right\}, R\right)$ is said to be a solution of PBVP (1.3) if and only if $y$ satisfies the dynamic equation

$$
y^{\Delta}(t)+p(t) y^{\sigma}(t)=f\left(t, y^{\sigma}(t)\right) \text {, everywhere on } J\left\{\mathrm{t}_{\mathrm{k}}\right\}, \mathrm{k}=1,2, \ldots, \mathrm{q},
$$

the impulsive condition

$$
\Delta y\left(t_{k}\right)=\gamma\left(t_{k}^{+}\right)-\gamma\left(t_{k}^{+}\right)=I_{k}\left(\gamma\left(t_{k}^{-}\right)\right), k=1,2, \ldots, q,
$$

and the periodic boundary condition

$$
y(0)=y(\sigma(T)) .
$$

Let $h: \mathbf{T} \rightarrow R$ be a rd-continuous function and consider the following periodic boundary value problem

$$
\left\{\begin{array}{l}
y^{\Delta}(t)+m(t) y^{\sigma}(t)=h(t), t \in J\left\{t_{k}\right\}, k=1,2, \ldots, q, \\
\Delta y\left(t_{k}\right)=-L_{k} y\left(t_{k}\right)+I_{k}\left(\eta\left(t_{k}\right)\right)+L_{k} \eta\left(t_{k}\right), k=1,2, \ldots, q, \\
\gamma(0)=\gamma(\sigma(T)) .
\end{array}\right.
$$

Lemma 3.1. $y \in P C$ is a solution of PBV P (3.1) if and only if

$$
\gamma(t)=\int_{0}^{\sigma(T)} G(t, s) h(s) \Delta s+\sum_{0<t_{k}<\sigma(T)} G\left(t, t_{k}\right)\left[-L_{k} \gamma\left(t_{k}\right)+I_{k}\left(\eta\left(t_{k}\right)\right)+L_{k} \eta\left(t_{k}\right)\right],
$$

where $\eta \in P C$ and

$$
G(t, s)= \begin{cases}\frac{e_{m}(s, t) e_{m}(\sigma(T), 0)}{e_{m}(\sigma(T),(0)-1}, & 0 \leq s \leq t \leq \sigma(T) \\ \frac{e_{m}(s, t)}{e_{m}(\sigma(T), 0)-1}, & 0 \leq t<s \leq \sigma(T)\end{cases}
$$

Proof. For the convenience of the reader, letting $w\left(t_{k}\right)=-L_{k} y\left(t_{k}\right)+I_{k}\left(\eta\left(t_{k}\right)\right)+L_{k} \eta\left(t_{k}\right)$, $k=1,2, \ldots, q$.

If $y$ is a solution of PBVP (3.1), for $t \in\left(0, t_{1}\right]$, we have

$$
\gamma(t)=e_{m}(0, t) \gamma(0)+\int_{0}^{t} e_{m}(s, t) h(s) \Delta s,
$$

and

$$
\gamma\left(t_{1}^{-}\right)=e_{m}\left(0, t_{1}\right) \gamma(0)+\int_{0}^{t_{1}} e_{m}\left(s, t_{1}\right) h(s) \Delta s .
$$

For any $t \in\left(t_{1}, t_{2}\right]$, in a similar way, we deduce that

$$
y(t)=e_{m}\left(t_{1}, t\right) y\left(t_{1}^{+}\right)+\int_{t_{1}}^{t} e_{m}(s, t) h(s) \Delta s
$$

From the fact that $\gamma\left(t_{1}^{+}\right)=\gamma\left(t_{1}^{-}\right)+w\left(t_{1}\right)$, this together with (3.4) and (3.5) yields that

$$
\gamma(t)=e_{m}(0, t) y(0)+\int_{0}^{t} e_{m}(s, t) h(s) \Delta s+e_{m}\left(t_{1}, t\right) w\left(t_{1}\right), t \in\left(t_{1}, t_{2}\right] .
$$


Repeating the above procession, for $t \in\left(t_{k}, t_{k+1}\right]$, it is not difficult to see that

$$
y(t)=e_{m}\left(t_{k}, t\right) y\left(t_{k}^{+}\right)+\int_{t_{k}}^{t} e_{m}(s, t) h(s) \Delta s
$$

with

$$
y\left(t_{k}^{-}\right)=e_{m}\left(0, t_{k}\right) y(0)+\int_{0}^{t_{k}} e_{m}\left(s, t_{k}\right) h(s) \Delta s+\sum_{0<t_{j}<t_{k}} e_{m}\left(t_{j}, t_{k}\right) w\left(t_{j}\right) .
$$

Consequently, it turns out that

$$
y(t)=e_{m}(0, t) y(0)+\int_{0}^{t} e_{m}(s, t) h(s) \Delta s+\sum_{0<t_{k}<t} e_{m}\left(t_{k}, t\right) w\left(t_{k}\right) \text { for all } t \in J .
$$

Since $y(0)=y(\sigma(T))$, then we have

$$
\gamma(0)=e_{m}(0, \sigma(T)) y(0)+\int_{0}^{\sigma(T)} e_{m}(s, \sigma(T)) h(s) \Delta s+\sum_{0<t_{k}<\sigma(T)} e_{m}\left(t_{k}, \sigma(T)\right) w\left(t_{k}\right),
$$

it follows

$$
\gamma(0)=\frac{1}{1-e_{m}(0, \sigma(T))}\left[\int_{0}^{\sigma(T)} e_{m}(s, \sigma(T)) h(s) \Delta s+\sum_{0<t_{k}<\sigma(T)} e_{m}\left(t_{k}, \sigma(T)\right) w\left(t_{k}\right)\right] .
$$

Substituting (3.8) into (3.7), we obtain

$$
\begin{aligned}
y(t)= & \frac{e_{m}(0, t)}{1-e_{m}(0, \sigma(T))}\left[\int_{0}^{\sigma(T)} e_{m}(s, \sigma(T)) h(s) \Delta s+\sum_{0<t_{k}<\sigma(T)} e_{m}\left(t_{k}, \sigma(T)\right) w\left(t_{k}\right)\right] \\
& +\left[\int_{0}^{t} e_{m}(s, t) h(s) \Delta s+\sum_{0<t_{k}<t} e_{m}\left(t_{k}, t\right) w\left(t_{k}\right)\right] \\
= & \int_{0}^{t}\left[\frac{e_{m}(0, t) e_{m}(s, \sigma(T))}{1-e_{m}(0, \sigma(T))}+e_{m}(s, t)\right] h(s) \Delta s+\int_{t}^{\sigma(T)} \frac{e_{m}(0, t) e_{m}(s, \sigma(T))}{1-e_{m}(0, \sigma(T))} h(s) \Delta s \\
& +\sum_{0<t_{k}<t}\left[\frac{e_{m}\left(0, t_{k}\right) e_{m}\left(t_{k}, \sigma(T)\right)}{1-e_{m}(0, \sigma(T))}+e_{m}\left(t_{k}, t\right)\right] w\left(t_{k}\right) \\
& +\sum_{t<t_{k}<\sigma(T)} \frac{e_{m}(0, t) e_{m}\left(t_{k}, \sigma(T)\right)}{1-e_{m}(0, \sigma(T))} w\left(t_{k}\right) \\
= & \int_{0}^{t} \frac{e_{m}(s, t) e_{m}(\sigma(T), 0)}{e_{m}(\sigma(T), 0)-1} h(s) \Delta s+\int_{t}^{\sigma(T)} \frac{e_{m}(s, t)}{e_{m}(\sigma(T), 0)-1} h(s) \Delta s \\
& +\sum_{0<t_{k}<t} \frac{e_{m}\left(t_{k}, t\right) e_{m}(\sigma(T), 0)}{e_{m}(\sigma(T), 0)-1} w\left(t_{k}\right)+\sum_{t<t_{k}<\sigma(T)} \frac{e_{m}\left(t_{k}, t\right)}{e_{m}(\sigma(T), 0)-1} w\left(t_{k}\right) \\
= & \int_{0}^{\sigma(T)} G(t, s) h(s) \Delta s+\sum_{0<t_{k}<\sigma(T)} G\left(t, t_{k}\right) w\left(t_{k}\right) .
\end{aligned}
$$


On the other hand, assume $y(t)$ satisfies (3.2) and notice that

$$
\begin{aligned}
& {\left[\int_{0}^{\sigma(T)} G(t, s) h(s) \Delta s\right]^{\Delta} } \\
= & \frac{1}{e_{m}(\sigma(T), 0)-1}\left[e_{m}(\sigma(T), 0) \int_{0}^{t} e_{m}(s, t) h(s) \Delta s+\int_{t}^{\sigma(T)} e_{m}(s, t) h(s) \Delta s\right] \\
= & \frac{e_{m}(\sigma(T), 0)}{e_{m}(\sigma(T), 0)-1}\left[\int_{0}^{t} e_{m}^{\Delta}(s, t) h(s) \Delta s+e_{m}(t, \sigma(t) h(t)]\right. \\
& +\frac{1}{e_{m}(\sigma(T), 0)-1}\left[\int_{t}^{\sigma(T)} e_{m}^{\Delta}(s, t) h(s) \Delta s-e_{m}(t, \sigma(t) h(t)]\right. \\
= & \ominus m \int_{0}^{\sigma(T)} G(t, s) h(s) \Delta s+h(t) .
\end{aligned}
$$

Analogously,

$$
\left[\sum_{0<t_{k}<\sigma(T)} G\left(t, t_{k}\right) w\left(t_{k}\right)\right]^{\Delta}=\ominus m \sum_{0<t_{k}<\sigma(T)} G\left(t, t_{k}\right) w\left(t_{k}\right) .
$$

As a result,

$$
\begin{aligned}
y^{\Delta}(t) & =\ominus m\left[\int_{0}^{\sigma(T)} G(t, s) h(s) \Delta s+\sum_{0<t_{k}<\sigma(T)} G\left(t, t_{k}\right) w\left(t_{k}\right)\right]+h(t) \\
& =\ominus m \gamma(t)+h(t) \\
& =-m(t) y^{\sigma}(t)+h(t) .
\end{aligned}
$$

Clearly, we have $y\left(t_{k}^{+}\right)-y\left(t_{k}^{-}\right)=w\left(t_{k}\right), y(0)=y(\sigma(T))$, for $k=1,2, \ldots, q$. The proof is completed.

Define an operator $F$ as follows:

$$
F x(t)=\int_{0}^{\sigma(T)} G(t, s) h(s) \Delta s+\sum_{0<t_{k}<\sigma(T)} G\left(t, t_{k}\right)\left[-L_{k} x\left(t_{k}\right)+I_{k}\left(\eta\left(t_{k}\right)\right)+L_{k} \eta\left(t_{k}\right)\right], t \in[0, \sigma(T)] .
$$

In view of $\left|G\left(t, t_{k}\right)\right| \leq\left|\frac{e_{m}(\sigma(T), 0)}{e_{m}(\sigma(T), 0)-1}\right|$, for $x, y \in P C$

$$
\begin{aligned}
|F x(t)-F y(t)| & \leq \sum_{0<t_{k}<\sigma(T)}\left|G\left(t, t_{k}\right) L_{k}\left[x\left(t_{k}\right)-\gamma\left(t_{k}\right)\right]\right| \\
& \leq\left|\frac{e_{m}(\sigma(T), 0)}{e_{m}(\sigma(T), 0)-1}\right| \sum_{k=1}^{m}\left|L_{k}\right| \cdot|| x-y|| .
\end{aligned}
$$

Under the condition of $\left|\frac{e_{m}(\sigma(T), 0)}{e_{m}(\sigma(T), 0)-1}\right| \sum_{k=1}^{q}\left|L_{k}\right|<1$, the PBVP (3.1) has one unique solution. 
Lemma 3.2. Suppose the following conditions hold

(i) The sequence $\left\{t_{k}\right\}_{k=1}^{q}$ satisfies $0 \leq t_{0}<t_{1}<\ldots<t_{q}$;

(ii) For $k=1,2, \ldots, q, t \geq t_{0}, 1-\mu(t) m(t)>0$, there are

$$
\begin{aligned}
& y^{\Delta}(t) \leq-m(t) y^{\sigma}(t), \\
& y\left(t_{k}^{+}\right) \leq b_{k} \gamma\left(t_{k}\right),
\end{aligned}
$$

where $b_{k}$ are constants and $b_{k}>0$.

Then

$$
\gamma(t) \leq \gamma\left(t_{0}\right) \prod_{t_{0}<t_{k}<t} b_{k} e_{\ominus m}\left(t, t_{0}\right)
$$

Proof. In view of (3.9), we have

$$
\left[e_{m}\left(t, t_{0}\right) y(t)\right]^{\Delta}=e_{m}\left(t, t_{0}\right) y^{\Delta}(t)+\gamma(\sigma(t)) e_{m}\left(t, t_{0}\right) m(t) \leq 0 .
$$

It means that $e_{m}\left(t, t_{0}\right) y(t)$ is a non-increasing on $\left[t_{0}, \sigma(T)\right]$. So we can easily get

$$
\gamma(t) \leq \gamma\left(t_{0}\right) e_{\ominus m}\left(t, t_{0}\right), t \in\left(t_{0}, t_{1}\right] .
$$

For $t \in\left(t_{1}, t_{2}\right]$, similarly, one can assert that

$$
\gamma(t) \leq \gamma\left(t_{1}^{+}\right) e_{\ominus m}\left(t, t_{1}\right) \leq b_{1} e_{\ominus m}\left(t, t_{1}\right) \gamma\left(t_{1}\right) \leq \gamma\left(t_{0}\right) b_{1} e_{\ominus m}\left(t, t_{0}\right) .
$$

Continuing with this procedure, we can get that for $t \in\left(t_{k}, t_{k+1}\right]$,

$$
\gamma(t) \leq \gamma\left(t_{k}^{+}\right) e_{\ominus m}\left(t, t_{k}\right) \leq b_{k} e_{\ominus m}\left(t, t_{k}\right) \gamma\left(t_{k}\right) \leq b_{1} b_{2} \cdots b_{k} \gamma\left(t_{0}\right) e_{\ominus m}\left(t, t_{0}\right),
$$

proceed inductively to obtain

$$
\gamma(t) \leq \gamma\left(t_{0}\right) \prod_{t_{0}<t_{k}<t} b_{k} e_{\ominus m}\left(t, t_{0}\right)
$$

The proof is completed.

Remark 3.1. If the inequalities of (3.9) and (3.10) are reversed, then the inequality in the conclusion is also reversed.

$$
\begin{aligned}
& \text { Lemma 3.3. Assume } \prod_{k=1}^{q}\left(1-L_{k}\right) e_{\ominus m}(\sigma(T), 0)<1, L_{k}<1 \text { and } \\
& y^{\Delta}(t) \leq-m(t) y^{\sigma}(t)-r_{y(t)} \\
& \Delta y\left(t_{k}\right) \leq-L_{k} y\left(t_{k}\right)-d_{y k} .
\end{aligned}
$$

Then, $y(t) \leq 0$ for all $t \in J$, where

$$
r_{y(t)}=\left\{\begin{array}{lr}
0, & y(0) \leq y(\sigma(T)), \\
\frac{m(t) \sigma(t)+1}{\sigma(T)}[y(0)-\gamma(\sigma(T))], & y(0)>\gamma(\sigma(T)),
\end{array}\right.
$$

and

$$
d_{y k}= \begin{cases}0, & y(0) \leq y(\sigma(T)), \\ \frac{L_{k} t_{k}}{\sigma(T)}[\gamma(0)-\gamma(\sigma(T))], & y(0)>\gamma(\sigma(T)) .\end{cases}
$$


Proof. Case 1: when $y(0) \leq y(\sigma(T))$, obviously, we have $r_{y(t)}=0, d_{y k}=0$. From Lemma 3.2, we see that,

$$
\gamma(t) \leq \gamma(0) \prod_{0<t_{k}<t}\left(1-L_{k}\right) e_{\ominus m}(t, 0) .
$$

Let $t=\sigma(T)$ to obtain

$$
\gamma(0) \leq \gamma(\sigma(T)) \leq \gamma(0) \prod_{0<t_{k}<\sigma(T)}\left(1-L_{k}\right) e_{\ominus m}(\sigma(T), 0) .
$$

It means

$$
\gamma(0)\left[1-\prod_{t_{0}<t_{k}<\sigma(T)}\left(1-L_{k}\right) e_{\ominus m}(\sigma(T), 0)\right] \leq 0,
$$

then $y(0) \leq 0$, returning to (3.15) we have $y(t) \leq 0, t \in J$.

Case 2: when $y(0)>y(\sigma \quad(T))$, define $\bar{y}(t)=y(t)+g(t)$, where $g(t)=\frac{t}{\sigma(T)}[\gamma(0)-\gamma(\sigma(T))], t \in J$. It is easy to check that $g(0)=0, \bar{y}(0)=\bar{\gamma}(\sigma(T))$ and $g(t) \geq 0$. Next, from (3.13) and (3.14), one can deduce that

$$
\begin{aligned}
\bar{y}^{\Delta}(t) & =\gamma^{\Delta}(t)+g^{\Delta}(t) \\
& \leq-m(t) y^{\sigma}(t)-r_{y(t)}+\frac{1}{\sigma(T)}[\gamma(0)-\gamma(\sigma(T))] \\
& =-m(t) y^{\sigma}(t)-m(t) g^{\sigma}(t) \\
& =-m(t) \bar{y}^{\sigma}(t), \\
\Delta \bar{y}\left(t_{k}\right) & =\Delta y\left(t_{k}\right)+\Delta g\left(t_{k}\right) \leq-L_{k} \gamma\left(t_{k}\right)-d_{y k}=-L_{k}\left[\gamma\left(t_{k}\right)-g\left(t_{k}\right)\right]=-L_{k} \bar{\gamma}\left(t_{k}\right) .
\end{aligned}
$$

In view of case 1 , one can easily see that $\bar{y}(t) \leq 0$, it means $y(t)+g(t) \leq 0$, this together with $g(t) \geq 0$ for $t \in J$ guarantees that $y(t) \leq 0, t \in J$. The proof is completed. 口

For $\alpha, \beta \in P C$, we write $\alpha \leq \beta$ if $\alpha(t) \leq \beta(t)$ for all $t \in J$. In such a case, we denote

$$
[\alpha, \beta]=\{y \in P C: \alpha(t) \leq \gamma(t) \leq \beta(t), \quad t \in J\} .
$$

Now, we are in the position to establish the main result.

Theorem 3.1. Assumed that the following conditions are satisfied

$\left(\mathrm{H}_{1}\right)$ There exist two functions : $\alpha, \beta \in P C \cap C^{1}\left(J \backslash\left\{t_{1}, t_{2}, \ldots, t_{q}\right\}, R\right) \alpha(t) \leq \beta(t)$ such that

$$
\left\{\begin{array}{l}
\alpha^{\Delta}(t)+p(t) \alpha^{\sigma}(t) \leq f\left(t, \alpha^{\sigma}(t)\right)-r_{\alpha(t)}, \quad t \in J, t \neq t_{k} \\
\Delta \alpha\left(t_{k}\right) \leq I_{k}\left(\alpha\left(t_{k}^{-}\right)\right)-d_{\alpha k}, \quad k=1,2, \ldots, q
\end{array}\right.
$$

and

$$
\left\{\begin{array}{l}
\beta^{\Delta}(t)+p(t) \beta^{\sigma}(t) \geq f\left(t, \beta^{\sigma}(t)\right)+r_{\beta(t)}, \quad t \in J, \quad t \neq t_{k}, \\
\Delta \beta\left(t_{k}\right) \geq I_{k}\left(\beta\left(t_{k}^{-}\right)\right)+d_{\beta k}, \quad k=1,2, \ldots, q,
\end{array}\right.
$$

where $1-\mu(t) m(t)>0, L_{k}<1, r_{\alpha(t)}, r_{\beta(t)}, d_{\alpha k}, d_{\beta k}$ are given by

$$
r_{\alpha(t)}=\left\{\begin{array}{lr}
0, & \alpha(0) \leq \alpha(\sigma(T)), \\
\frac{m(t) \sigma(t)+1}{\sigma(T)}[\alpha(0)-\alpha(\sigma(T))], & \alpha(0)>\alpha(\sigma(T)),
\end{array}\right.
$$




$$
\begin{aligned}
& d_{\alpha k}= \begin{cases}0, & \alpha(0) \leq \alpha(\sigma(T)), \\
\frac{L_{k} t_{k}}{\sigma(T)}[\alpha(0)-\alpha(\sigma(T))], & \alpha(0)>\alpha(\sigma(T)),\end{cases} \\
& r_{\beta(t)}= \begin{cases}0, & \beta(0) \geq \beta(\sigma(T)), \\
\frac{m(t) \sigma(t)+1}{\sigma(T)}[\beta(\sigma(T))-\beta(0)], & \beta(0)<\beta(\sigma(T)),\end{cases} \\
& d_{\beta k}=\left\{\begin{array}{lr}
0, & \beta(0) \geq \beta(\sigma(T)), \\
\frac{L_{k} t_{k}}{\sigma(T)}[\beta(\sigma(T))-\beta(0)], & \beta(0)<\beta(\sigma(T)),
\end{array}\right.
\end{aligned}
$$

that is, $\alpha(t)$ and $\beta(t)$ are lower and upper solutions of PBV P (13), respectively; $\left(\mathrm{H}_{2}\right) f(t, x): \mathbf{T} \times R \rightarrow R$ is $r d$ - continuous at the first variable and continuous at the second variable such that

$$
f\left(t, \gamma^{\sigma}(t)\right)-f\left(t, x^{\sigma}(t)\right) \geq[p(t)-m(t)]\left(\gamma^{\sigma}(t)-x^{\sigma}(t)\right),
$$

for $\alpha(t) \leq x(t) \leq y(t) \leq \beta(t)$;

$\left(\mathrm{H}_{3}\right) I_{k} \in C(R, R)$ satisfies

$$
I_{k}\left(\gamma\left(t_{k}\right)\right)-I_{k}\left(x\left(t_{k}\right)\right) \geq-L_{k}\left(\gamma\left(t_{k}\right)-x\left(t_{k}\right)\right)
$$

for $\alpha(t) \leq x\left(t_{k}\right) \leq y\left(t_{k}\right) \leq \beta(t), k=1,2, \cdots, q, 0 \leq L_{k}<1$;

$\left(\mathrm{H}_{4}\right)$ There exists $m(t) \in C(R, R)$ satisfying $1-\mu(t) m(t)>0$ and

$$
\prod_{k=1}^{q}\left(1-L_{k}\right) e_{\ominus m}(\sigma(T), 0)<1,\left|\frac{e_{m}(\sigma(T), 0)}{e_{m}(\sigma(T), 0)-1}\right| \sum_{k=1}^{q} L_{k}<1 .
$$

Then, there exist monotone sequences $\alpha_{n}(t)$ and $\beta_{n}(t)$ with $\alpha_{0}=\alpha, \beta_{0}=\beta$ such that

$$
\lim _{n \rightarrow \infty} \alpha_{n}(t)=\gamma_{*}(t), \lim _{n \rightarrow \infty} \beta_{n}(t)=\gamma^{*}(t),
$$

uniformly on $J$, and $y_{*}(t), y_{*}(t)$ are the minimal and maximal solutions of $P B V P$ (1.3) such that $y_{*} \leq y \leq y_{*}$, where $y$ is any solution of PBV P (1.3) satisfying $\alpha \leq y \leq \beta$ on J.

Proof. For any $\eta \in[\alpha, \beta]$, consider the PBVP (3.1) with $h(t)=f(t, \eta \sigma(t))+[m(t)-p$ $(t)] \eta^{\sigma}(t)$. The condition $\left(\mathrm{H}_{4}\right)$ and Lemma 3.1 guarantee that PBVP (3.1) has a unique solution $y(t)$. Define the operator $A:[\alpha, \beta] \rightarrow P C$ such that $y(t)=A \eta$. Next, we show that the operator $A$ satisfies the two properties as follows:

(i) $\alpha \leq A \alpha, A \beta \leq \beta$;

(ii) $A$ is a monotone nondecreasing function, i.e. for any $\eta_{1}, \eta_{2} \in[\alpha, \beta], \eta_{1} \leq \eta_{2}$ implies $A \eta_{1} \leq A \eta_{2}$.

To see (i), letting $\bar{\gamma}=\alpha-\alpha_{1}$, where $\alpha_{1}=A \alpha$. Owing to the condition $\left(\mathrm{H}_{1}\right)$ and (3.1), we have

$$
\begin{aligned}
\bar{y}^{\Delta}(t) & =\alpha^{\Delta}(t)-\alpha_{1}^{\Delta}(t) \\
& \leq f\left(t, \alpha^{\sigma}(t)\right)-r_{\alpha(t)}-p(t) \alpha^{\sigma}(t)-\left[-m(t) \alpha_{1}^{\sigma}(t)+f\left(t, \alpha^{\sigma}(t)\right)+(m(t)-p(t)) \alpha^{\sigma}(t)\right] \\
& =-r_{\alpha(t)}-m(t)\left[\alpha^{\sigma}(t)-\alpha_{1}^{\sigma}(t)\right] \\
& =-r_{\bar{y}(t)}-m(t) \bar{y}^{\sigma}(t)
\end{aligned}
$$




$$
\begin{aligned}
\Delta \bar{y}\left(t_{k}\right) & =\Delta \alpha\left(t_{k}\right)-\Delta \alpha_{1}\left(t_{k}\right) \\
& \leq I_{k}\left(\alpha\left(t_{k}\right)\right)-d_{\alpha k}-\left[-L_{k} \alpha_{1}\left(t_{k}\right)+I_{k}\left(\alpha\left(t_{k}\right)\right)+L_{k} \alpha\left(t_{k}\right)\right] \\
& =-d_{\alpha k}-L_{k}\left[\alpha\left(t_{k}\right)-\alpha_{1}\left(t_{k}\right)\right] \\
& =-d_{\alpha k}-L_{k} \bar{\gamma}\left(t_{k}\right) .
\end{aligned}
$$

By virtue of Lemma 3.3, we achieve $\bar{y}(t) \leq 0$ on $J$, i.e. $\alpha \leq A \alpha$. Similarly, we can show that $A \beta \leq \beta$.

To see (ii), let $\bar{\gamma}=y_{1}-y_{2}$, where $y_{1}=A \eta_{1}, y_{2}=A \eta_{2}$. Applying the condition $\left(\mathrm{H}_{2}\right)$ and $\left(\mathrm{H}_{3}\right)$ together with (3.1) to obtain that

$$
\begin{aligned}
\bar{y}^{\Delta}(t)= & y_{1}^{\Delta}(t)-y_{2}^{\Delta}(t) \\
= & f\left(t, \eta_{1}^{\sigma}(t)\right)+(m(t)-p(t)) \eta_{1}^{\sigma}(t)-m(t) \gamma_{1}^{\sigma}(t)-f\left(t, \eta_{2}^{\sigma}(t)\right)-(m(t)-p(t)) \eta_{2}^{\sigma}(t) \\
& +m(t) y_{2}^{\sigma}(t) \\
\leq & -m(t)\left[\gamma_{1}^{\sigma}(t)-\gamma_{2}^{\sigma}(t)\right]+f\left(t, \eta_{1}^{\sigma}(t)\right)-f\left(t, \eta_{2}^{\sigma}(t)\right)+(m(t)-p(t))\left(\eta_{1}^{\sigma}(t)-\eta_{2}^{\sigma}(t)\right) \\
\leq & -m(t) \bar{y}^{\sigma}(t), \\
\Delta \bar{y}\left(t_{k}\right)= & \Delta y_{1}\left(t_{k}\right)-\Delta y_{2}\left(t_{k}\right) \\
= & -L_{k} \gamma_{1}\left(t_{k}\right)+I_{k}\left(\eta_{1}\left(t_{k}\right)\right)+L_{k} \eta_{1}\left(t_{k}\right)-\left[-L_{k} y_{2}\left(t_{k}\right)+I_{k}\left(\eta_{2}\left(t_{k}\right)\right)+L_{k} \eta_{2}\left(t_{k}\right)\right] \\
\leq & -L_{k} \bar{y}\left(t_{k}\right)+I_{k}\left(\eta_{1}\left(t_{k}\right)\right)-I_{k}\left(\eta_{2}\left(t_{k}\right)\right)+L_{k}\left[\eta_{1}\left(t_{k}\right)-\eta_{2}\left(t_{k}\right)\right] \\
\leq & -L_{k} \bar{y}\left(t_{k}\right) .
\end{aligned}
$$

The Lemma 3.3 yields that $\bar{\gamma}(t) \leq 0$ on $J$, i.e. $A \eta_{1} \leq A \eta_{2}$.

Now, define the sequences $\alpha_{n+1}=A \alpha_{n}, \beta_{n+1}=A \beta_{n}$ with $\alpha_{0}=\alpha, \beta_{0}=\beta$. Notice that

$$
\begin{aligned}
& \alpha_{n}=\int_{0}^{\sigma(T)} G(t, s) h_{n-1}(s) \Delta s+\sum_{0<t_{k}<\sigma(T)}\left[-L_{k} \alpha_{n}\left(t_{k}\right)+I_{k}\left(\alpha_{n-1}\left(t_{k}\right)\right)+L_{k} \alpha_{n-1}\left(t_{k}\right)\right], \\
& \beta_{n}=\int_{0}^{\sigma(T)} G(t, s) \bar{h}_{n-1}(s) \Delta s+\sum_{0<t_{k}<\sigma(T)}\left[-L_{k} \beta_{n}\left(t_{k}\right)+I_{k}\left(\beta_{n-1}\left(t_{k}\right)\right)+L_{k} \beta_{n-1}\left(t_{k}\right)\right],
\end{aligned}
$$

where

$$
\begin{aligned}
& h_{n-1}(t)=f\left(t, \alpha_{n-1}^{\sigma}(t)\right)+[m(t)-p(t)] \alpha_{n-1}^{\sigma}(t), t \in[0, \sigma(T)], \\
& \bar{h}_{n-1}(t)=f\left(t, \beta_{n-1}^{\sigma}(t)\right)+[m(t)-p(t)] \beta_{n-1}^{\sigma}(t), t \in[0, \sigma(T)] .
\end{aligned}
$$

The two properties of operator A guarantee that the sequences $\alpha_{n}$ and $\beta_{n}$ satisfy

$$
\alpha=\alpha_{0} \leq \alpha_{1} \leq \cdots \leq \alpha_{n} \leq \cdots \leq \beta_{n} \leq \cdots \leq \beta_{1} \leq \beta_{0}=\beta .
$$

Consequently, there exist $y *(t)$ and $y^{*}(t)$ such that

$$
\lim _{n \rightarrow \infty} \alpha_{n}(t)=\gamma_{*}(t), \text { and } \lim _{n \rightarrow \infty} \beta_{n}(t)=\gamma^{*}(t),
$$

uniformly on $J$. It is obviously seen that $y_{*}(t)$ and $y^{*}(t)$ satisfy PBVP (1.3).

Let $y(t)$ be any solution of PBVP (1.3) such that $y \in[\alpha, \beta]$. Assuming that there exists a positive integer $n$ such that $\alpha_{n}(t) \leq y(t) \leq \beta_{n}(t)$, since $A$ is a monotone nondecreasing operator, we obtain $\alpha_{n+1}(t)=A \alpha_{n}(t) \leq A y(t)=y(t), \beta_{n+1}(t)=A \beta_{n}(t) \geq A y(t)=$ $y(t)$, furthermore, it follows $\alpha_{n+1}(t) \leq y(t) \leq \beta_{n+1}(t)$. In view of $\alpha_{0}(t) \leq y(t) \leq \beta_{0}(t)$, 
proceed inductively to obtain $\alpha_{n}(t) \leq y(t) \leq \beta_{n}(t)$ for any positive integer $n$. Therefore $y_{*}$ $(t) \leq y(t) \leq y^{*}(t)$ on $J$ as $n \rightarrow+\infty$.

The proof is completed.

Remark 3.2. Observe that Theorem 3.1 generalize the Theorem 3.1 of Geng et al. [6]. When $p(t)=t, \sigma(t)=t, t \in[0, T] \cap \mathbf{T}, t \neq t_{k}, k=1,2, \ldots, q$, the PBVP (1.3) becomes to the PBVP (1.2) which has been investigated in [6], moreover, we have the following corollary if we set $m(t)=M$.

Corollary 3.1 ([6]). Suppose the following conditions :

$\left(\mathrm{H}_{5}\right)$ The functions $\alpha, \beta$ are lower and upper solutions of PBV P (1.2) respectively, such that $\alpha(t) \leq \beta(t)$ for $t \in J$;

$\left(\mathrm{H}_{6}\right) f(t, y): \mathbf{T} \times R \rightarrow R$ is $r d$-continuous at the first variable and continuous at the second variable such that

$$
f(t, y)-f(t, x) \geq-M(y-x), \text { for } \alpha(t) \leq x(t) \leq \gamma(t) \leq \beta(t) ;
$$

$\left(\mathrm{H}_{7}\right) I_{k} \in C(R, R)$ satisfies

$$
I_{k}\left(\gamma\left(t_{k}\right)\right)-I_{k}\left(x\left(t_{k}\right)\right) \geq-L_{k}\left(\gamma\left(t_{k}\right)-x\left(t_{k}\right)\right)
$$

for $\alpha(t) \leq x\left(t_{k}\right) \leq y\left(t_{k}\right) \leq \beta(t), k=1,2, \ldots, q, 0 \leq L_{k}<1$;

$\left(\mathrm{H}_{8}\right)$ There exists a constant $M$ satisfying $\mu(t)<\frac{1}{M}$ and $0 \leq L_{k}<1$ such that

$$
\frac{1}{1-e_{-M}(\sigma(T), 0)} \sum_{k=1}^{q} L_{k}<1, \quad \prod_{k=1}^{q}\left(1-L_{k}\right) e_{-M}(\sigma(T), 0)<1 .
$$

Then, there exist monotone sequences $\alpha_{n}(t)$ and $\beta_{n}(t)$ with $\alpha_{0}=\alpha, \beta_{0}=\beta$ such that

$$
\lim _{n \rightarrow \infty} \alpha_{n}(t)=\gamma_{*}(t), \quad \lim _{n \rightarrow \infty} \beta_{n}(t)=\gamma^{*}(t),
$$

uniformly on $J$, and $y *(t), y^{*}(t)$ are the minimal and the maximal solutions of PBV P (1.2), respectively, such that

$$
\alpha=\alpha_{0} \leq \alpha_{1} \leq \alpha_{2} \leq \cdots \leq \alpha_{n} \leq \gamma_{*} \leq \gamma \leq \gamma^{*} \leq \beta_{n} \leq \cdots \leq \beta_{2} \leq \beta_{1} \leq \beta_{0}=\beta \text { on } \mathrm{J}
$$

where $y$ is any solution of PBVP (1.2) satisfying $\alpha(t) \leq y(t) \leq \beta(t)$ on $J$.

\section{Lower and upper solutions in the reversed order}

In this section, we obtain the existence criterion of extremal solutions for PBVP (1.3) under another case: $\alpha \geq \beta$, where $\alpha$ and $\beta$ are lower and upper solutions of PBVP (1.3).

Assume $h: \mathbf{T} \rightarrow R$ is rd-continuous, we consider the following periodic boundary value problem:

$$
\left\{\begin{array}{l}
y^{\Delta}(t)-m(t) y^{\sigma}(t)=h(t), t \in J \backslash\left\{t_{k}\right\}, \quad k=1,2, \ldots, q, \\
\Delta y\left(t_{k}\right)=L_{k} \gamma\left(t_{k}\right)+I_{k}\left(\eta\left(t_{k}\right)\right)-L_{k} \eta\left(t_{k}\right), \quad k=1,2, \ldots, q, \\
\gamma(0)=\gamma(\sigma(T)) .
\end{array}\right.
$$

Lemma 4.1. $y \in P C$ is a solution of PBVP (4.1) if and only if

$$
y(t)=\int_{0}^{\sigma(T)} \bar{G}(t, s) h(s) \Delta s+\sum_{0<t_{k}<\sigma(T)} \bar{G}\left(t, t_{k}\right)\left[L_{k} y\left(t_{k}\right)+I_{k}\left(\eta\left(t_{k}\right)\right)-L_{k} \eta\left(t_{k}\right)\right]
$$


where

$$
\bar{G}(t, s)= \begin{cases}\frac{e_{-m}(s, t) e_{-m}(\sigma(T), 0)}{e_{-m}(\sigma(T), 0)-1}, & 0 \leq s \leq t \leq \sigma(T), \\ \frac{e_{-m}(s, t)}{e_{-m}(\sigma(T), 0)-1}, & 0 \leq t<s \leq \sigma(T) .\end{cases}
$$

Proof. The detailed proof is very similar to one given in Lemma 3.1. Here, we omit it for brevity.

Similarly, define an operator $\bar{F}$ :

$$
\bar{F} \gamma(t)=\int_{0}^{\sigma(T)} \bar{G}(t, s) h(s) \Delta s+\sum_{0<t_{k}<\sigma(T)} \bar{G}\left(t, t_{k}\right)\left[L_{k} \gamma\left(t_{k}\right)+I_{k}\left(\eta\left(t_{k}\right)\right)-L_{k} \eta\left(t_{k}\right)\right], \quad t \in[0, \sigma(T)] .
$$

Lemma 4.2. Assume $\prod_{k=1}^{q}\left(1+L_{k}\right) e_{\ominus-m}(\sigma(T), 0)<1, L_{k}>-1$ and

$$
\begin{aligned}
& y^{\Delta}(t) \geq m(t) y^{\sigma}(t)+\bar{r}_{y(t)}, \\
& \Delta y\left(t_{k}\right) \geq L_{k} \gamma\left(t_{k}\right)+\bar{d}_{y k} .
\end{aligned}
$$

Then, $y(t) \leq 0$ for all $t \in J$, where

$$
\bar{r}_{\gamma(t)}=\left\{\begin{array}{lr}
0, & \gamma(\sigma(T)) \leq \gamma(0), \\
\frac{m(t)[\sigma(T)-\sigma(t)]+1}{\sigma(T)}[\gamma(\sigma(T))-\gamma(0)], & \gamma(\sigma(T))>\gamma(0),
\end{array}\right.
$$

and

$$
\bar{d}_{y k}=\left\{\begin{array}{lr}
0, & y(\sigma(T)) \leq y(0), \\
\frac{L_{k}\left(\sigma(T)-t_{k}\right)}{\sigma(T)}[y(\sigma(T))-\gamma(0)], & y(\sigma(T))>\gamma(0) .
\end{array}\right.
$$

Proof. Following the similar lines as in Lemma 3.3, we can prove this Lemma. The detailed process is omitted here for brevity.

Theorem 4.1. Assume that

$\left(\mathrm{G}_{1}\right)$ There exist two functions : $\alpha, \beta \in P C \cap C^{1}\left(J \backslash\left\{t_{1}, t_{2}, \ldots, t_{q}\right\}, R\right), \beta(t) \leq \alpha(t)$ such that

$$
\left\{\begin{array}{l}
\alpha^{\Delta}(t)+p(t) \alpha^{\sigma}(t) \leq f\left(t, \alpha^{\sigma}(t)\right)-\bar{r}_{\alpha(t),} \quad t \in J, t \neq t_{k} \\
\Delta \alpha\left(t_{k}\right) \leq I_{k}\left(\alpha\left(t_{k}^{-}\right)\right)-\bar{d}_{\alpha k}, \quad k=1,2, \ldots, q,
\end{array}\right.
$$

and

$$
\left\{\begin{array}{l}
\beta^{\Delta}(t)+p(t) \beta^{\sigma}(t) \geq f\left(t, \beta^{\sigma}(t)\right)+\bar{r}_{\beta(t)}, \quad t \in J, \quad t \neq t_{k}, \\
\Delta \beta\left(t_{k}\right) \geq I_{k}\left(\beta\left(t_{k}^{-}\right)\right)+\bar{d}_{\beta k}, \quad k=1,2, \ldots, q,
\end{array}\right.
$$

where $1-\mu(t) m(t)>0, L_{k}>-1, \bar{r}_{\alpha(t)}, \bar{r}_{\beta(t)}, \bar{d}_{\alpha k}, \bar{d}_{\beta k}$ are given by

$$
\begin{aligned}
& \bar{r}_{\alpha(t)}=\left\{\begin{array}{lr}
0, & \alpha(0) \leq \alpha(\sigma(T)), \\
\frac{m(t)[\sigma(T)-\sigma(t)]+1}{\sigma(T)}[\alpha(0)-\alpha(\sigma(T))], & \alpha(0)>\alpha(\sigma(T)),
\end{array}\right. \\
& \bar{d}_{\alpha k}= \begin{cases}0, & \alpha(0) \leq \alpha(\sigma(T)), \\
\frac{L_{k}\left[\sigma(T)-t_{k}\right]}{\sigma(T)}[\alpha(0)-\alpha(\sigma(T))], & \alpha(0)>\alpha(\sigma(T)),\end{cases}
\end{aligned}
$$




$$
\begin{aligned}
& \bar{r}_{\beta(t)}=\left\{\begin{array}{lr}
0, & \beta(0) \geq \beta(\sigma(T)), \\
\frac{m(t)[\sigma(T)-\sigma(t)]+1}{\sigma(T)}[\beta(\sigma(T))-\beta(0)], & \beta(0)<\beta(\sigma(T)),
\end{array}\right. \\
& \bar{d}_{\beta k}=\left\{\begin{array}{lr}
0, & \beta(0) \geq \beta(\sigma(T)), \\
\frac{L_{k}\left[\sigma(T)-t_{k}\right]}{\sigma(T)}[\beta(\sigma(T))-\beta(0)], & \beta(0)<\beta(\sigma(T)),
\end{array}\right.
\end{aligned}
$$

that is, $\alpha(t)$ and $\beta(t)$ are lower and upper solutions of PBV P (1.3), respectively;

$\left(\mathrm{G}_{2}\right) f(t, x): \mathbf{T} \times R \rightarrow R$ is $r d$-continuous at the first variable and continuous at the second variable such that

$$
f\left(t, y^{\sigma}(t)\right)-f\left(t, x^{\sigma}(t)\right) \leq[p(t)+m(t)]\left(\gamma^{\sigma}(t)-x^{\sigma}(t)\right),
$$

for $\beta(t) \leq x(t) \leq y(t) \leq \alpha(t)$;

$\left(\mathrm{G}_{3}\right) I_{k} \in C(R, R)$ satisfies

$$
I_{k}\left(y\left(t_{k}\right)\right)-I_{k}\left(x\left(t_{k}\right)\right) \leq L_{k}\left(y\left(t_{k}\right)-x\left(t_{k}\right)\right)
$$

for $\beta(t) \leq x\left(t_{k}\right) \leq y\left(t_{k}\right) \leq \alpha(t), k=1,2, \ldots, q, 0 \leq L_{k}<1$;

$\left(\mathrm{G}_{4}\right)$ There exists $m(t)$ satisfying $1-\mu(t) m(t)>0$ and

$$
\prod_{k=1}^{q}\left(1+L_{k}\right) e_{\ominus-m}(\sigma(T), 0)>1, \quad \frac{1}{\left|e_{-m}(\sigma(T), 0)-1\right|} \sum_{k=1}^{q} L_{k}<1 .
$$

Then, there exist monotone sequences $\alpha_{n}(t)$ and $\beta_{n}(t)$ with $\alpha_{0}=\alpha, \beta_{0}=\beta$ such that

$$
\lim _{n \rightarrow \infty} \beta_{n}(t)=\gamma_{*}(t), \quad \lim _{n \rightarrow \infty} \alpha_{n}(t)=\gamma^{*}(t),
$$

uniformly on $J$, and $y *(t), y^{*}(t)$ are the minimal and maximal solutions of $P B V P(1.3)$ such that $y_{*} \leq y \leq y^{*}$, where $y$ is any solution of PBV P (1.3) satisfying $\beta \leq y \leq \alpha$ on J.

Proof. Setting $h(t)=f\left(t, \eta^{\sigma}(t)\right)-[m(t)+p(t)] \eta^{\sigma}(t), t \in J$, by using Lemma 4.1 and Lemma 4.2 coupled with the monotone iterative technique, we can prove this result by imitating the proof of Theorem 3.1. $\square$

\section{Examples}

In this section, we provide two examples to illustrate the feasibility and applicability of our obtained results. Under the case of lower and upper solutions with reverse-order, the first one is devoted to the existence of extremal solutions to PBVP (5.1) on a time scale defined as the real numbers set, and in the second one, the existence of extremal solutions to PBVP (5.5) on a time scale defined as a sequence of discrete points union a closed real interval is guaranteed.

Example 5.1. Let $\mathbf{T}=R$ and consider the following periodic boundary value problem

$$
\left\{\begin{array}{l}
y^{\Delta}(t)=\sin \gamma(t)-4 y(t)+e^{t}, t \in\left[0, \frac{1}{3}\right] \cap \mathbf{T}, t \neq \frac{1}{5} \\
\Delta y\left(\frac{1}{5}\right)=\frac{1}{30} \\
y(0)=\gamma\left(\frac{1}{3}\right)
\end{array}\right.
$$

In this case, when $x \leq y, x, y \in R$, we have

$$
f(t, y)-f(t, x)=\sin y-\sin x-4(y-x) \leq 3(y-x), \text { for all } t \in\left[0, \frac{1}{3}\right],
$$

then, the condition $\left(\mathrm{G}_{2}\right)$ is satisfied for $m(t)=3$. 
Taking $L_{1}=\frac{1}{3}$ to verify $\left(G_{4}\right)$, it is easy to check that,

$$
\frac{1}{1-e_{-m}(\sigma(T), 0)} \sum_{k=1}^{m} L_{k}=\frac{e}{3(e-1)}<1, \quad \prod_{k=1}^{m}\left(1+L_{k}\right) e_{\ominus-m}(\sigma(T), 0)=\frac{4}{3} e>1 .
$$

It follows that the condition $\left(\mathrm{G}_{4}\right)$ is valid.

Since $I_{k}=\frac{1}{30}$, this together with $L_{1}=\frac{1}{3}$ guarantees that the condition $\left(\mathrm{G}_{3}\right)$ holds.

Let

$$
\alpha(t)=e^{t}-\frac{\pi}{10}, \quad \beta(t)=\left\{\begin{array}{l}
0.46, t \in\left[0, \frac{1}{5}\right] \\
0.5, t \in\left(\frac{1}{5}, \frac{1}{3}\right]
\end{array}\right.
$$

Due to the fact that $\alpha(0)<\alpha\left(\frac{1}{3}\right)$, then $\bar{r}_{\alpha}=\bar{d}_{\alpha}=0$.

$$
\begin{aligned}
& f(t, \alpha(t))-\alpha^{\Delta}(t)=\sin \left(e^{t}-\frac{\pi}{10}\right)+4\left(e^{t}-\frac{\pi}{10}\right), \\
& \min _{t \in\left[0, \frac{1}{3}\right]} f(t, \alpha(t))-\alpha^{\Delta}(t)=\sin \left(1-\frac{\pi}{10}\right)+4\left(1-\frac{\pi}{10}\right)>0 .
\end{aligned}
$$

Then, we have

$$
\alpha^{\Delta}(t) \leq f(t, \alpha(t))-\bar{r}_{\alpha}(t), t \in\left[0, \frac{1}{3}\right]
$$

and

$$
\Delta \alpha\left(\frac{1}{5}\right)=0 \leq \frac{1}{30},
$$

it follows that $\alpha$ is a lower solution of PBVP (5.1).

On the other hand, $\beta(0)=0.46<0.50=\beta\left(\frac{1}{3}\right)$, then $\bar{r}_{\beta}=0.24-0.36 t, \bar{d}_{\beta}=\frac{2}{375}$. By virtue of $\beta^{\Delta}(t)=0$ for $t \in\left[0, \frac{1}{3}\right] \backslash\left\{\frac{1}{5}\right\}$, it follows that

$$
\begin{aligned}
& f(t, \beta(t))+\bar{r}_{\beta}=\sin (0.46)-4 \times 0.46+e^{t}+0.24-0.36 t \leq-0.00664913<0, t \in\left[0, \frac{1}{5}\right), \\
& f(t, \beta(t))+\bar{r}_{\beta}=\sin (0.5)-4 \times 0.5+e^{t}+0.24-0.36 t \leq-0.00496204<0, t \in\left(\frac{1}{5}, \frac{1}{3}\right]
\end{aligned}
$$

and

$$
\Delta \beta\left(\frac{1}{5}\right)=0.04 \geq \frac{1}{30}+\frac{2}{375},
$$

which implies that $\beta$ is an upper solution of PBVP (5.1), and $\beta \leq \alpha$ for $t \in\left[0, \frac{1}{3}\right]$. As a result, Theorem 4.1 guarantees there exist monotone sequences that approximate the extremal solutions of PBVP (5.1).

Example 5.2. Consider the following periodic boundary value problem on time scale

$$
\left\{\begin{array}{l}
x^{\Delta}(t)-2 x^{\sigma}(t)=3 \sin x^{\sigma}(t)+e^{t}, t \in\left[0, \frac{1}{3}\right] \cap \mathbf{T}, t \neq \frac{1}{5}, \\
\Delta y\left(\frac{1}{5}\right)=\frac{1}{15} \\
y(0)=\gamma\left(\frac{1}{3}\right)
\end{array}\right.
$$

where $\mathbf{T}=\left\{\frac{1}{5}-\left(\frac{1}{5}\right)^{N}\right\} \cup\left[\frac{1}{5}, \frac{1}{3}\right], N=\{1,2, \ldots\}$.

In this case, $p(t)=-2, f\left(t, x^{\sigma}(t)\right)=3 \sin x^{\sigma}(t)+e^{t}, t \in\left[0, \frac{1}{3}\right] \cap \mathbf{T}, t \neq \frac{1}{5}$. Since

$$
f\left(t, y^{\sigma}(t)\right)-f\left(t, x^{\sigma}(t)\right)=3 \sin \gamma^{\sigma}(t)-3 \sin x^{\sigma}(t) \leq 3\left(y^{\sigma}(t)-x^{\sigma}(t)\right)
$$


for all $x \leq y, x, y \in R, t \in\left[0, \frac{1}{3}\right]$, which shows the condition $\left(\mathrm{G}_{2}\right)$ is satisfied for $m(t)$ $=3$.

Since $I_{k}=\frac{1}{15}$, then the condition $\left(\mathrm{G}_{3}\right)$ holds naturally.

Taking $L_{1}=\frac{1}{3}$ to calculate

$$
\frac{1}{1-e_{-m}(\sigma(T), 0)} \sum_{k=1}^{m} L_{k}=\frac{e}{3(e-1)}<1, \quad \prod_{k=1}^{m}\left(1+L_{k}\right) e_{\ominus-m}(\sigma(T), 0)=\frac{4}{3} e>1,
$$

which implies that condition $\left(\mathrm{G}_{4}\right)$ is true.

Next, we show that

$$
\alpha(t)=0, \beta(t)= \begin{cases}-0.7, t \in\left[0, \frac{1}{5}\right] \cap \mathbf{T}, \\ -0.6, t \in\left(\frac{1}{5}, \frac{1}{3}\right] \cap \mathbf{T}\end{cases}
$$

are a pair of lower and upper solutions of PBVP (5.5) which satisfy $\beta \leq \alpha$ for all $t \in\left[0, \frac{1}{3}\right] \cap \mathbf{T}$.

we may see that $\alpha(0)<\alpha\left(\frac{1}{3}\right)$, thus $\bar{r}_{\alpha(t)}=\bar{d}_{\alpha k}=0$. Note that $\alpha^{\Delta}(t)=0$ for $\alpha^{\Delta}(t)=0<e^{t}=f(t, \alpha(t))-p(t) \alpha^{\sigma}(t)-\bar{r}_{\alpha(t)}$, $\alpha^{\Delta}(t)=0<e^{t}=f(t, \alpha(t))-p(t) \alpha^{\sigma}(t)-\bar{r}_{\alpha(t)}$ and $\Delta \alpha\left(\frac{1}{5}\right)=0<\frac{1}{15}$, which yields $\alpha$ is a lower solution of PBVP (5.5).

On the other hand, $\beta(0)=-0.7<-0.6=\beta\left(\frac{1}{3}\right)$ implies

$$
\bar{r}_{\beta(t)}=\left\{\begin{array}{l}
\frac{6-9 t}{10}, t \in\left[\frac{1}{5}, \frac{1}{3}\right], \\
\frac{114}{250}-\frac{9 t}{50}, t \in\left\{\frac{1}{5}-\left(\frac{1}{5}\right)^{N}\right\},
\end{array} \quad \bar{d}_{\beta k}=\frac{1}{75},\right.
$$

therefore

$$
\begin{aligned}
& f\left(t, \beta^{\sigma}(t)\right)+\bar{r}_{\beta}(t)-p(t) \beta^{\sigma}(t)-\beta^{\Delta}(t)=3 \sin (-0.6)-1.2+e^{t}+\frac{6-9 t}{10} \leq-1.19831<0, t \in\left(\frac{1}{5}, \frac{1}{3}\right], \\
& f\left(t, \beta^{\sigma}(t)\right)+\bar{r}_{\beta}(t)-p(t) \beta^{\sigma}(t)-\beta^{\Delta}(t)=3 \sin (-0.7)-1.4+e^{t}+\frac{114}{250}-\frac{9 t}{50} \leq-1.69125<0, t \in\left\{\frac{1}{5}-\left(\frac{1}{5}\right)^{N}\right\},
\end{aligned}
$$

and

$$
\Delta \beta\left(\frac{1}{5}\right)=\frac{1}{10} \geq \frac{1}{15}+\frac{1}{75} .
$$

Consequently, $\beta$ is an upper solution of PBVP (5.5). In view of Theorem 4.1, there exist monotone sequences that approximate the extremal solutions of PBVP (5.5).

\section{Acknowledgement}

This work was supported by the National Natural Science Foundation of China(Grant Nos. 11071108, 10761007) and the Provincial Natural Science Foundation of Jiangxi, China (Grant Nos. 2010GZS0147, 2007GZS2051).

\section{Authors' contributions}

CZ conceived the main idea of the study, XZ carried out the main parts of the draft. CZ give many valuable suggestions and corrected the main theorems in the discussion. All authors read and approved the final manuscript.

\section{Competing interests}

The authors declare that they have no competing interests.

Received: 7 October 2011 Accepted: 7 June 2012 Published: 7 June 2012

\section{References}

1. Lakshmikantham, V, Bainov, DD, Simeonov, PS: Theory of Impulsive Differential Equations. World Scientific, Singapore (1989) 
2. Bainov, DD, Simeonov, PS: Impulsive Differential Equations: Periodic Solutions and Applications. Longman Scientific and Technical, Harlow (1993)

3. Ding, $\mathrm{W}, \mathrm{Mi}, \mathrm{J}, \mathrm{Han}, \mathrm{M}$ : Periodic boundary value problems for the first order impulsive functional differential equations. Appl Math Comput. 165, 433-446 (2005). doi:10.1016/.amc.2004.06.022

4. Li, JL, Shen, JH: Periodic boundary value problems for delay differential equations with impulses. J Comput Appl Math. 193, 563-573 (2006). doi:10.1016/j.cam.2005.05.037

5. Aulbach, B, Hilger, S: Linear dynamical processes with inhomogeneous time scale. Nonlinear Dynamics and Quantum Dynamical Systems. Akademie, Berlin (1990)

6. Geng, FJ, Xu, YC, Zhu, DM: Periodic boundary value problems for first-order impulsive dynamic equations on time scales. Nonlinear Anal. 69, 4074-4087 (2008). doi:10.1016/j.na.2007.10.038

7. Agarwal, RP, Bohner, M, O'Regan, D, Peterson, A: Dynamic equations on time scales: a survey. J Comput Appl Math 141, 1-26 (2002). doi:10.1016/S0377-0427(01)00432-0

8. Lakshmikantham, V, Sivasundaram, S, Kaymakcalan, B: Dynamic Systems on Measure Chains. Kluwer, Dordrecht (1996)

9. Bohner, M, Peterson, A: Dynamic equations on time scales: An Introduction with Applications. Birkhäuser, Boston (2001)

10. Chang, YK, Li, WT: Existence results for impulsive dynamic equations on time scales with nonlocal initial conditions. Math Comput Model. 43, 377-384 (2006). doi:10.1016/j.mcm.2005.12.015

11. Wang, DB: Positive solutions for nonlinear periodic boundary value problems of impulsive dynamic equations on time scales. Comput Math Appl. 56, 1496-1504 (2008). doi:10.1016/j.camwa.2008.02.038

12. Benchohra, M, Ntouyas, SK, Ouahab, A: Extremal solutions of second order impulsive dynamic equations on time scale. J Math Anal Appl. 324, 425-434 (2006). doi:10.1016/j.jmaa.2005.12.028

13. Chen, HB, Wang, HH: Triple positive solutions for boundary value problem for p-Laplacian impulsive dynamic equations on time scales. Math Comput Model. 47, 917-924 (2008). doi:10.1016/j.mcm.2007.06.012

14. He, ZM: Existence of two solutions of $m$-point boundary value problem for second order dynamic equations on time scales. J Math Anal Appl. 296, 97-109 (2004). doi:10.1016/j.jmaa.2004.03.051

15. Song, CX, Xiao, CT: Positive solutions for p-Laplacian functional dynamic equations on time scales. Nonlinear Anal. 66, 1989-1998 (2007). doi:10.1016/j.na.2006.02.036

16. Sun, HR, Li, WT: Positive solutions of second-order half-linear dynamic equations on time scales. Appl Math Comput. 158, 331-344 (2004). doi:10.1016/j.amc.2003.08.089

17. Espinar, VO, Vivero, DR: Existence and approximation of extremal solutions to first-order infinite systems of functional dynamic equations. J Math Anal Appl. 339, 590-597 (2008). doi:10.1016/j.jmaa.2007.06.031

18. Guo, D, Lakshmikantham, V: Nonlinear Problems in Abstract Cones. Academic Press, New York (1988)

19. Avery, Rl, Chyan, CJ, Henderson, J: Twin solutions of a boundary value problems for ordinary differential equations and finite difference equations. Comput Math Appl. 42, 695-704 (2001). doi:10.1016/50898-1221(01)00188-2

20. Zhu, CX: Research on some problems for nonlinear operators. Nonlinear Anal. 71, 568-4571 (2009)

21. Zhu, CX, Chen, CF: Calculations of random fixed point index. J Math Anal Appl. 339, 839-844 (2008). doi:10.1016/j. jmaa.2007.07.040

22. Zhu, CX, Yin, JD: Calculations of a random fixed point index of a random semi-closed 1-set-contractive operator. Math Comput Model. 51, 1135-1139 (2010). doi:10.1016/j.mcm.2009.12.023

23. He, ZM, Zhang, XM: Monotone iterative technique for first order impulsive difference equations with periodic boundary conditions. Appl Math Comput. 156, 605-620 (2004). doi:10.1016/j.amc.2003.08.013

24. Wang, WB, Yang, XX, Shen, JH: Boundary value problems involving upper and lower solutions in reverse order. J Comput Appl Math. 230, 1-7 (2009). doi:10.1016/j.cam.2008.10.040

25. Cabada, A, Otero-Espinar, V, Pouso, RL: Existence and approximation of solutions for first-order discontinuous difference equations with nonlinear global conditions in the presence of lower and upper solutions. Comput Math Appl. 39, 21-33 (2000). doi:10.1016/S0898-1221(99)00310-7

doi:10.1186/1687-1847-2012-76

Cite this article as: Zhang and Zhu: Periodic boundary value problems for first order dynamic equations on time scales. Advances in Difference Equations 2012 2012:76.

\section{Submit your manuscript to a SpringerOpen ${ }^{\ominus}$ journal and benefit from:}

- Convenient online submission

- Rigorous peer review

- Immediate publication on acceptance

- Open access: articles freely available online

- High visibility within the field

- Retaining the copyright to your article

Submit your next manuscript at $\boldsymbol{s p r i n g e r o p e n . c o m ~}$ 